\title{
Astragaloside IV combating liver cirrhosis through the PI3K/Akt/mTOR signaling pathway
}

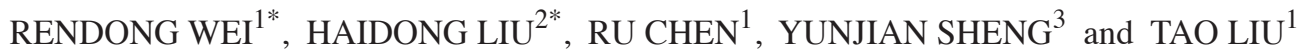 \\ Departments of ${ }^{1}$ Hepatology and ${ }^{2}$ Digestive Diseases, The Sixth People's Hospital of Qingdao, \\ Qingdao, Shandong 266033; ${ }^{3}$ Department of Infectious Diseases, The Affiliated Hospital \\ of Southwest Medical University, Luzhou, Sichuan 646000, P.R. China
}

Received April 19, 2018; Accepted October 23, 2018

DOI: $10.3892 /$ etm.2018.6966

\begin{abstract}
Astragaloside IV (AS-IV) in improving liver cirrhosis injury in rats and its effect on the phosphoinositide 3-kinase (PI3K)/protein kinase B (Akt)/mechanistic target of rapamycin (mTOR) signaling pathway were observed. Rat model of liver cirrhosis was induced by injection of carbon tetrachloride $\left(\mathrm{CCl}_{4}\right)$. A total of 36 Sprague-Dawley (SD) rats were randomly divided into three groups: the normal control group $(n=10)$, the model control group $(n=13)$, and the AS-IV group $(n=13)$. The normal control group was injected with olive oil and given carboxymethyl cellulose (CMC)-Na (10 $\mathrm{ml} / \mathrm{kg} /$ day), the model control group was given CMC-Na (10 $\mathrm{ml} / \mathrm{kg} /$ day), and the AS-IV group underwent intragastric administration of AS-IV (20 ml/kg/day). The content of alanine transaminase (ALT) and aspartate transaminase (AST) of rats was detected. The levels of tumor necrosis factor- $\alpha$ (TNF- $\alpha$ ), interleukin (IL)- 6 and IL- $1 \beta$ in serum were detected via enzyme-linked immunosorbent assay (ELISA). Hematoxylin and eosin (H\&E) staining was applied to observe morphological changes in liver tissues. The expression of collagens in liver tissues was detected via Masson's trichrome staining. Additionally, the expression of proteins in liver tissues was detected via western blotting. Compared with those in the blank group, the levels of AST, ALT, TNF- $\alpha$, IL-6 and IL-1 $\beta$ were higher, the expression level of collagens in liver tissues was increased, and the expression ratios of phosphorylated (p)-PI3K/PI3K, p-Akt/Akt and p-mTOR/mTOR proteins were increased in the model group. Compared with the model group, AS-IV could significantly decrease the content of AST, ALT, TNF- $\alpha$, IL- 6 and IL- $\beta$ in serum
\end{abstract}

Correspondence to: Dr Tao Liu, Department of Hepatology, The Sixth People's Hospital of Qingdao, 9 Fushun Road, Qingdao, Shandong 266033, P.R. China

E-mail: liutaoxyy@163.com

*Contributed equally

Key words: astragaloside IV, liver cirrhosis, inflammatory cytokine, $\mathrm{PI} 3 \mathrm{~K} / \mathrm{Akt} / \mathrm{mTOR}$ signaling pathway of rats, obviously inhibit the expression of collagens in liver tissues and decrease the expression ratios of $\mathrm{p}-\mathrm{PI} 3 \mathrm{~K} / \mathrm{PI} 3 \mathrm{~K}$, $\mathrm{p}-\mathrm{Akt} / \mathrm{Akt}$ and $\mathrm{p}-\mathrm{mTOR} / \mathrm{mTOR}$ proteins in liver tissues. AS-IV can inhibit the inflammatory response so as to reduce the expression of collagens, and its mechanism may play a key role by inhibiting the PI3K/Akt/mTOR signaling pathway.

\section{Introduction}

Liver cirrhosis is a common chronic liver disease in China, which is characterized by diffuse fibrosis as well as pseudo-lobular and nodular formation. According to statistics, patients with liver cirrhosis account for $\sim 10 \%$ of the total number of patients in China (1). A study has revealed that the pathogenic factors of liver cirrhosis include hepatitis viruses, drugs and poisons, cholestasis, metabolism and inheritance, schistosomiasis and alcohol. These pathogenic factors stimulate liver cells for a long time, induce the activation of hepatic stellate cells, and make the generated extracellular matrices more than degraded ones, thus resulting in the extracellular matrix deposition and liver tissue remodeling (2).

The phosphoinositide 3-kinase (PI3K)/protein kinase $\mathrm{B}$ (Akt)/mechanistic target of rapamycin (mTOR) signal transduction pathway can promote cell growth, proliferation, differentiation and invasion, and it also plays key roles in anti-apoptosis, immune regulation and pro-angiogenesis. In addition, a study has evidenced that this pathway exerts crucial effects on the occurrence and development of liver fibrosis (3). Inflammation is a key factor triggering liver fibrosis and simulating the progression of liver cirrhosis (4). PI3K and mTOR can upregulate anti-inflammatory cytokines, inhibit the expression of pro-inflammatory cytokines, the inflammatory response of liver cells, and the proliferation and invasion of hepatic stellate cells, and promote the degradation of the liver extracellular matrix (5).

A study has demonstrated that astragaloside IV (AS-IV), one of the active components of Astragalus saponins in Astragalus membranaceus, has various pharmacological effects such as antioxidation, anti-inflammation and anti-infection (6). AS-IV can alleviate the inflammatory damage induced by orthotopic liver transplantation in rats through inhibiting the transcriptional activity of nuclear factor $\kappa$-light-chain-enhancer of activated B cells (NF- $\mathrm{B}$ ) (7). According to another study, 
AS-IV pretreatment can reduce cerebral ischemia-reperfusion injury in rats, which is mainly due to its ability to inhibit neutrophil adhesion-associated molecules, thereby inhibiting inflammation (8). Recent studies have manifested that AS-IV pretreatment can protect kidney injury, and its mechanisms include reducing inflammation and inhibiting apoptosis (9). In the present study, a rat model of liver cirrhosis was established to investigate whether AS-IV pretreatment can protect rats from liver cirrhosis and the effect of AS-IV on the PI3K/Akt/mTOR signaling pathway, providing basic research on the prevention and treatment of liver cirrhosis using AS-IV.

\section{Materials and methods}

Materials and reagents. AS-IV (Aladdin Reagent Co., Ltd., Shanghai, China), alanine transaminase (ALT) and aspartate transaminase (AST) detection kits, as well as interleukin (IL)-6, IL-1 $\beta$ and tumor necrosis factor- $\alpha$ (TNF- $\alpha$ ) (cat. nos. H052, H007, H002; all from Nanjing Jiancheng Bioengineering Institute, Nanjing, China), rabbit anti-rat phosphorylated (p)-PI3K, PI3K, p-Akt, Akt, p-mTOR, mTOR and glyceraldehyde 3-phosphate dehydrogenase (GAPDH) polyclonal antibodies, secondary goat anti-rabbit polyclonal antibody (cat. nos. bs-6417R, bs-10657R, bs-3043R, bs-0115R, bs-5329R, bs-1992R, bs-0755R, bs-0295G; all from BIOSS, Beijing, China) and Masson's staining solution (Wuhan Sanying Biotechnology, Wuhan, China), and hematoxylin and eosin (H\&E) staining kit, bicinchoninic acid (BCA) protein quantitation kit and tissue lysate (all from Beyotime Institute of Biotechnology, Nantong, China).

Experimental animal grouping and treatment. A Sprague-Dawley (SD) male rat model (4 months of age, 210-240 g; Beijing Vital River Laboratory Animal Technology Co., Ltd., Beijing, China) of liver cirrhosis was induced by the intraperitoneal injection of $50 \%$ carbon tetrachloride $\left(\mathrm{CCl}_{4}\right)$ (the volume ratio of $\mathrm{CCl}_{4}$ to olive oil was $\left.1: 1\right)$ twice a week for 8 weeks. A total of $36 \mathrm{SD}$ rats were randomly divided into three groups: the normal control group $(n=10)$, the model control group $(n=13)$, and the AS-IV group $(n=13)$. The rats were kept in a cage with controlled temperature and light conditions $\left(24^{\circ} \mathrm{C}\right.$ and $12 / 12$ light cycles) and had free access to water and food. The humidity was $60 \pm 10 \%$. The normal control group was intraperitoneally injected with olive oil twice a week, and given intragastric administration of $0.5 \%$ carboxymethyl cellulose (CMC)-Na (10 ml/kg/day); the model control group was given intragastric administration of $0.5 \%$ CMC-Na (10 ml/kg/day) during modeling; and the AS-IV group underwent the intragastric administration of AS-IV (20 $\mathrm{ml} / \mathrm{kg} / \mathrm{day})$ during modeling. The rats were sacrificed at the end of the 8th week, and underwent solid instead of liquid fasting overnight on the day before the end of the experiment. The next day, the rats were anesthetized, and their abdominal venous blood and liver were taken. After the blood was placed at room temperature and centrifuged at 2,600 $\mathrm{x}$ g for $10 \mathrm{~min}$, the supernatant serum was carefully drawn and stored at $-80^{\circ} \mathrm{C}$ for standby application. After the liver was weighed, the left lobe liver tissues were fixed in formalin for the histopathological study, and the remaining liver tissues were stored at $-80^{\circ} \mathrm{C}$ for western blotting. The study was approved by the Ethics Committee of the Sixth People's Hospital of Qingdao (Qingdao, China)

Determination of biochemical indexes in serum of rats. The activities of AST and ALT in serum of rats in each group were detected via AST and ALT kits, and the content of TNF- $\alpha$, IL- 6 and IL-1 $\beta$ in serum of rats in each group was detected using enzyme-linked immunosorbent assay (ELISA). All the operations were carried out according to the manufacturer's instructions.

Detection of the liver histopathology of rats. The fixed liver tissues in each group were selected. These tissues underwent dehydration and transparency at first, immersed in wax and embedded into wax blocks next, and cut into blank slices finally. After that, H\&E staining and Masson's trichrome staining were carried out based on the histopathological conventional methods. After the stained slices were dehydrated with gradient alcohol and made transparent via xylene, they were sealed with gum. Then the histopathological changes in the liver of rats in each group were observed using a microscope (Olympus Corp., Tokyo, Japan).

Detection of the expression of proteins relevant to the PI3K/Akt/mTOR signaling pathway via western blotting. About $50 \mathrm{mg}$ of rat liver tissues frozen at $-80^{\circ} \mathrm{C}$ were taken from each group. After the total protein was extracted with the tissue lysate, the protein concentration was determined via $\mathrm{BCA}$ assay, and then the proteins were mixed with the loading buffer and underwent thermal denaturation. A total of $40 \mu \mathrm{g}$ proteins were taken from each group for separation by $15 \%$ sodium dodecyl sulfate-polyacrylamide gel electrophoresis (SDS-PAGE). Then the membrane was transferred through wet processes, and the separated proteins were electrotransferred onto a polyvinylidene difluoride (PVDF) membrane. The blocking solution bovine serum albumin (BSA) was adopted for blocking for $2 \mathrm{~h}$ at room temperature, and primary antibodies including p-PI3K, PI3K, p-Akt, Akt, p-mTOR, mTOR and GAPDH (diluted at 1:1,000) were added for incubation in a refrigerator at $4^{\circ} \mathrm{C}$ overnight, followed by membrane washing with Tris-buffered saline with Tween-20 (TBST) for 3 times. Afterwards, secondary antibody (diluted at 1:2,000) were added for incubation at room temperature for $2 \mathrm{~h}$. After the membrane washing with TBST for 3 times, color development was conducted using the enhanced chemiluminescent (ECL) developing solution (Thermo Fisher Scientific, Inc., Waltham, MA, USA), and records were scanned via a gel imager (Bio-Rad Laboratories, Inc., Hercules, CA, USA). The densitometry of the blots was performed using ImageJ software (National Institutes of Health, Bethesda, MD, USA).

Statistical analysis. Statistical Product and Service Solutions (SPSS) 17.0 software (IBM Corp., Armonk, NY, USA) was applied for data processing, and the data were expressed as mean \pm standard deviation. The t-test was implemented for the intergroup comparison. ANOVA was used for comparison between multiple groups and LSD test was the post hoc test. $\mathrm{P}<0.05$ was considered to indicate a statistically significant difference. 

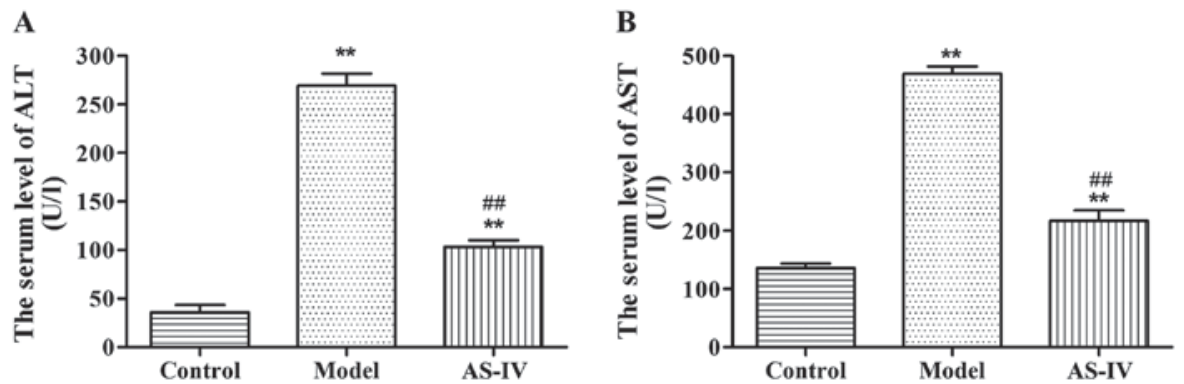

Figure 1. Effects of AS-IV on the content of (A) ALT and (B) AST in serum of rats. ${ }^{* *} \mathrm{P}<0.01$ vs. control group. ${ }^{\# \#} \mathrm{P}<0.01$ vs. model group. AS-IV, astragaloside IV; ALT, alanine transaminase; AST, aspartate transaminase.

A

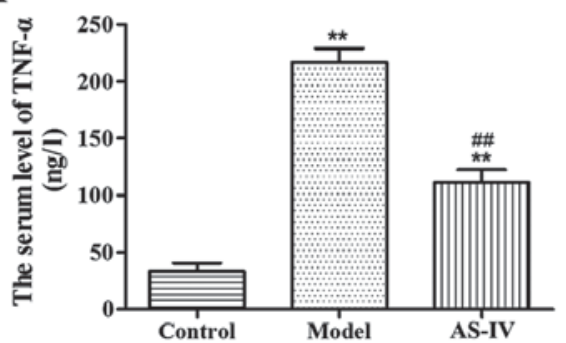

B

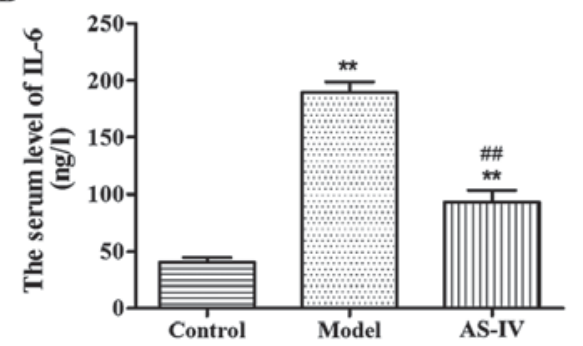

C

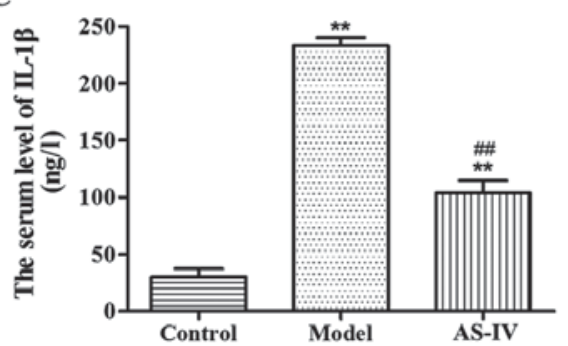

Figure 2. Effects of AS-IV on the content of (A) TNF- $\alpha$, (B) IL-6 and (C) IL-1 $\beta$ in serum of rats. ${ }^{* *} \mathrm{P}<0.01$ vs. control group. ${ }^{\# /} \mathrm{P}<0.01$ vs. model group. AS-IV, astragaloside IV; TNF- $\alpha$, tumor necrosis factor- $\alpha$; IL, interleukin.
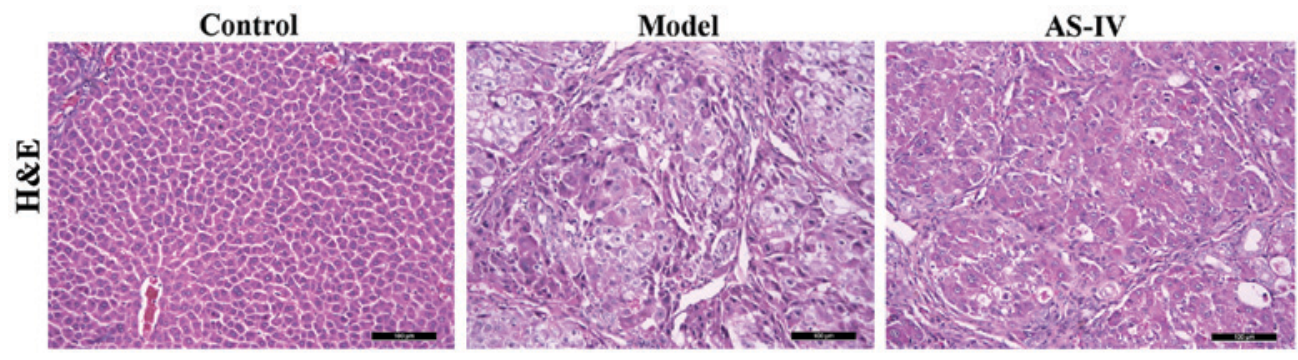

Figure 3. Detection of the effect of AS-IV on the liver histopathology of rats via H\&E staining. AS-IV, astragaloside IV; H\&E, hematoxylin and eosin.

\section{Results}

Effects of AS-IV on AST and ALT in serum of rats. Compared with those in the normal control group, the levels of AST and ALT in serum of rats in the model group were significantly increased $(\mathrm{p}<0.01)$. Compared with those in the model group, the levels of AST and ALT in serum of rats in the AS-IV administration group were remarkably decreased $(\mathrm{p}<0.01)$, indicating that the degree of rat liver injury in the AS-IV administration group was lowered than that in the model group (Fig. 1).

Effects of AS-IV on inflammatory cytokines in serum of rats. Compared with those in the normal control group, the content of TNF- $\alpha$, IL- 6 and IL-1 $\beta$ in serum of rats in the model group was obviously increased $(\mathrm{p}<0.01)$. Compared with those in the model group, the expression levels of TNF- $\alpha$, IL-6 and IL-1 $\beta$ significantly declined in serum of rats in the AS-IV group $(p<0.01)$, indicating that the inflammatory response of rats in the AS-IV group was reduced compared with that of rats in the model group (Fig. 2).
Effect of AS-IV on the liver histopathology of rats. There were intact liver lobule structures and central veins in the liver of rats in the normal control group. Destructed liver structures and inflammatory cell infiltration appeared in the liver of rats in the model group, while the above symptoms of rat liver tissues in the AS-IV treatment group were remarkably improved (Fig. 3).

Effect of AS-IV on the expression of rat collagens. Compared with the blank control group, obvious collagen hyperplasia was observed in liver tissues of the model group, and the fiber rope formed was relatively thick; whereas collagen deposition in liver tissues in the AS-IV treatment group was significantly reduced compared with that of the model group, and the fiber rope formed was relatively thin (Fig. 4).

Effect of AS-IV on the expression of proteins relevant to the PI3K/Akt/mTOR signaling pathway in liver tissues of rats. Compared with those in the control group, the expression levels of $\mathrm{p}-\mathrm{PI} 3 \mathrm{~K} / \mathrm{PI} 3 \mathrm{~K}, \mathrm{p}-\mathrm{Akt} / \mathrm{Akt}$, and $\mathrm{p}-\mathrm{mTOR} / \mathrm{mTOR}$ proteins 

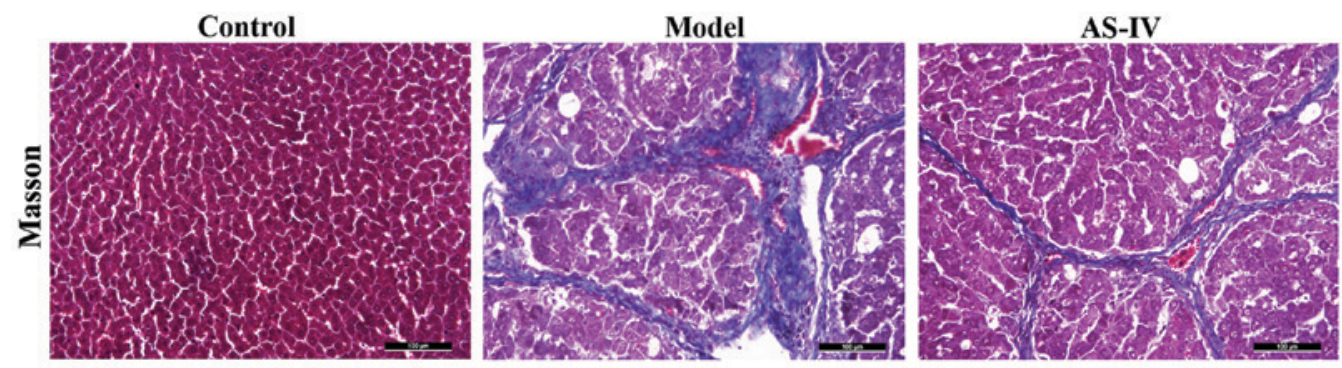

Figure 4. Detection of the effect of AS-IV on the expression of collagens in liver tissues of rats via Masson's trichrome staining. AS-IV, astragaloside IV.
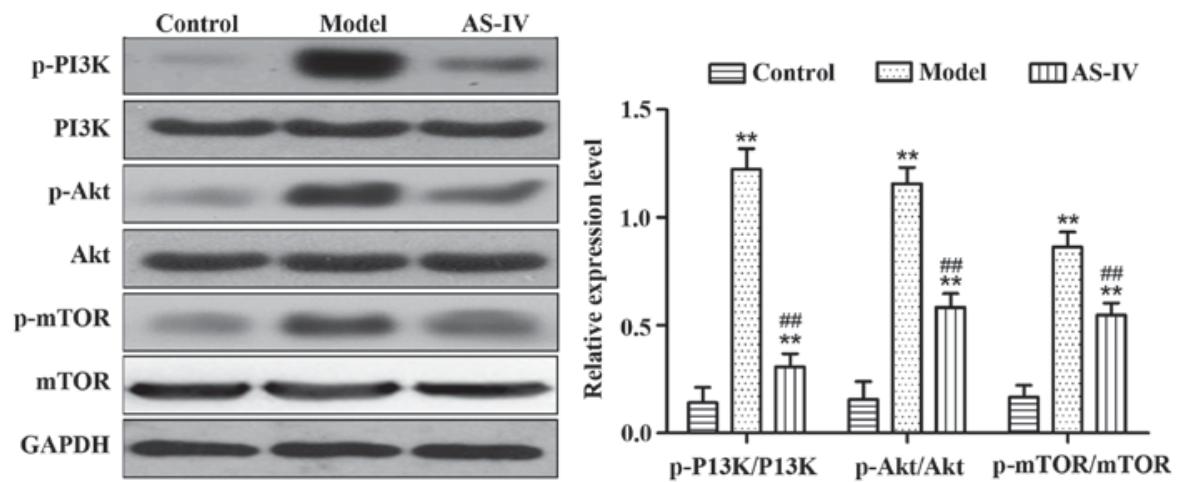

Figure 5. Detection of the expression of proteins relevant to the PI3K/Akt/mTOR signaling pathway in liver tissues via western blotting. ${ }^{* *} \mathrm{P}<0.01$ vs. control group. ${ }^{\# /} \mathrm{P}<0.01$ vs. model group. PI3K, phosphoinositide 3-kinase; mTOR, mechanistic target of rapamycin.

in liver tissues of the model group significantly increased $(\mathrm{p}<0.01)$. Compared with the model group, AS-IV obviously reduced the expression of $\mathrm{p}-\mathrm{PI} 3 \mathrm{~K} / \mathrm{PI} 3 \mathrm{~K}, \mathrm{p}-\mathrm{Akt} / \mathrm{Akt}$, and $\mathrm{p}-\mathrm{mTOR} / \mathrm{mTOR}$ proteins in rat liver tissues $(\mathrm{p}<0.01)$ (Fig. 5).

\section{Discussion}

Liver cirrhosis results from many chronic liver diseases developing to the late stage. The long-term stimulation of damage factors leads to diffuse necrosis of liver cells, and promotes the proliferation of fibrous tissues and the regeneration of nodular hepatocytes, thus ultimately contributing to the remodeling of liver lobule structures and blood vessels (10). A large number of basic and clinical studies have manifested that treatments can reverse the progression of liver fibrosis $(11,12)$. A great controversy currently exists in the treatment of liver cirrhosis, and some studies have revealed that early treatment can reverse liver cirrhosis (13-16).

PI3K phosphorylates phosphoinositides via the phosphorylation of serine 308 and threonine 473 by phosphoinositide-dependent kinase-1 (17). PI3 on the inositol ring generates phosphatidylinositol 4,5-bisphosphate $\left(\mathrm{PIP}_{2}\right)$ and phosphatidylinositol $(3,4,5)$-trisphosphate $\left(\mathrm{PIP}_{3}\right)$, and then acts as the second messenger in cells. Akt acts as a serine/threonine protein kinase, and can bind to $\mathrm{PIP}_{2}$ and $\mathrm{PIP}_{3}$ under the action of the phosphatidylinositol-dependent protein kinase, which promotes the transfer of Akt from the cytoplasm to the cell membrane (18). According to a study, platelet-derived growth factors can induce the phosphorylation of PI3K and Akt. Activated Akt can promote the phosphorylation of downstream substrates such as mTOR, Bad and the caspase family, having a variety of biological effects (19). Inhibiting the PI3K/Akt signaling pathway can obviously reduce the proliferation of hepatic stellate cells and the expression levels of type I collagen messenger ribonucleic acid (mRNA) and protein (20).

$\mathrm{mTOR} / \mathrm{p} 70 \mathrm{~S} 6 \mathrm{~K}$ protein plays a crucial role in the activation process of hepatic stellate cells (21). Inhibiting the activation of mTOR/p70S6K protein, a downstream target of the PI3K/Akt signaling pathway, can inhibit the proliferation and activation of hepatic stellate cells as well as the synthesis and secretion of collagens (22). This study showed that low-dose rapamycin can suppress the progression of liver fibrosis, whose mechanism is to inhibit the activation of mTOR/p70S6 so as to suppress the activation of hepatic fibroblasts (17). Another study has revealed that the mTOR inhibitors, sirolimus and everolimus, can significantly slow the development of liver fibrosis, and inhibit the migration of hepatic stellate cells and the production of collagens (23).

In the present study, the rat model of liver cirrhosis was established through the intraperitoneal injection of $\mathrm{CCl}_{4}$, and then the protective role of AS-IV intervention in the liver of rats and its effect on the PI $3 \mathrm{~K} / \mathrm{Ak} / \mathrm{mTOR}$ signaling pathway were investigated. Compared with those in the normal control group, the levels of AST, ALT, TNF- $\alpha$, IL- 6 and IL- $1 \beta$ in serum of rats in the model group were obviously increased. Compared with those in the model group, the levels of TNF- $\alpha$, IL- 6 and IL- $1 \beta$ significantly declined in serum of rats in the AS-IV group, indicating that the liver injury degree and inflammatory response of rats in the AS-IV group were lower than those in the model group. The histopathological detection revealed that there were intact liver lobule structures and central veins in the liver of rats in the normal control group. Destructed liver 
structures, inflammatory cell infiltration, and the significant increase in collagen expression appeared in the liver of rats in the model group, while the above symptoms of rat liver tissues in the AS-IV treatment group were remarkably improved, and collagen deposition in liver tissues in the AS-IV treatment group was significantly reduced compared with that in the model group. Based on western blotting results, compared with those in the control group, the expression levels of $\mathrm{p}-\mathrm{PI} 3 \mathrm{~K} / \mathrm{PI} 3 \mathrm{~K}$, $\mathrm{p}-\mathrm{Akt} / \mathrm{Akt}$, and $\mathrm{p}-\mathrm{mTOR} / \mathrm{mTOR}$ proteins in rat liver tissues in the model group increased significantly. AS-IV obviously reduced the expression ratios of $\mathrm{p}-\mathrm{PI} 3 \mathrm{~K} / \mathrm{PI} 3 \mathrm{~K}$, p-Akt/Akt, and $\mathrm{p}-\mathrm{mTOR} / \mathrm{mTOR}$ proteins in rat liver tissues, thus inhibiting the $\mathrm{PI} 3 \mathrm{~K} / \mathrm{Akt} / \mathrm{mTOR}$ signaling pathway.

In summary, $\mathrm{AS}$-IV protects $\mathrm{CCl}_{4}$-induced liver cirrhosis, and its mechanism may play a role in inhibiting the PI3K/Akt $/ \mathrm{mTOR}$ signaling pathway. This study provides a clear research basis for the treatment of liver cirrhosis with AS-IV in clinic.

\section{Acknowledgements}

Not applicable.

\section{Funding}

No funding was received.

\section{Availability of data and materials}

The datasets used and/or analyzed during the current study are available from the corresponding author on reasonable request.

\section{Authors' contributions}

RW and HL wrote the manuscript and conducted the experimental animal grouping and treatment. RC analyzed the biochemical indexes. YS and TL contributed to western blotting. All authors read and approved the final manuscript.

\section{Ethics approval and consent to participate}

The study was approved by the Ethics Committee of the Sixth People's Hospital of Qingdao (Qingdao, China).

\section{Patient consent for publication}

Not applicable.

\section{Competing interests}

The authors declare that they have no competing interests.

\section{References}

1. Liaw YF, Lin DY, Chen TJ and Chu CM: Natural course after the development of cirrhosis in patients with chronic type B hepatitis: A prospective study. Liver 9: 235-241, 1989.

2. Hsu YS, Chien RN, Yeh CT, Sheen IS, Chiou HY, Chu CM and Liaw YF: Long-term outcome after spontaneous HBeAg seroconversion in patients with chronic hepatitis B. Hepatology 35: 1522-1527, 2002.

3. Zhang Y and Liu YP: Research advances in PI3K/Akt signaling pathway and tumor multidrug resistance. Zhonghua Linchuang Yishi Zazhi 5: 446-449, 2011.
4. Pettinelli P, Obregón AM and Videla LA: Molecular mechanisms of steatosis in nonalcoholic fatty liver disease. Nutr Hosp 26: 441-450, 2011.

5. Weichhart T and Säemann MD: The PI3K/Akt/mTOR pathway in innate immune cells: Emerging therapeutic applications. Ann Rheum Dis 67 (Suppl 3): iii70-iii74, 2008.

6. Wang SG, Xu Y, Chen JD, Yang CH and Chen XH: Astragaloside IV stimulates angiogenesis and increases nitric oxide accumulation via JAK2/STAT3 and ERK1/2 pathway. Molecules 18: 12809-12819, 2013.

7. Cheng MX, Chen ZZ, Cai YL, Liu CA and Tu B: Astragaloside IV protects against ischemia reperfusion in a murine model of orthotopic liver transplantation. Transplant Proc 43: 1456-1461, 2011.

8. Li M, Qu YZ, Zhao ZW, Wu SX, Liu YY, Wei XY, Gao L and Gao GD: Astragaloside IV protects against focal cerebral ischemia/reperfusion injury correlating to suppression of neutrophils adhesion-related molecules. Neurochem Int 60 : 458-465, 2012.

9. Xin Y, Li G, Liu H and Ai D: AS-IV protects against kidney IRI through inhibition of NF- $\kappa$ B activity and PUMA upregulation. Int J Clin Exp Med 8: 18293-18301, 2015.

10. Pinzani M, Rosselli M and Zuckermann M: Liver cirrhosis. Best Pract Res Clin Gastroenterol 25: 281-290, 2011.

11. Lee UE and Friedman SL: Mechanisms of hepatic fibrogenesis. Best Pract Res Clin Gastroenterol 25: 195-206, 2011.

12. Hernandez-Gea V and Friedman SL: Pathogenesis of liver fibrosis. Annu Rev Pathol 6: 425-456, 2011.

13. Chang TT, Liaw YF, Wu SS, Schiff E, Han KH, Lai CL, Safadi R, Lee SS, Halota W, Goodman Z, et al: Long-term entecavir therapy results in the reversal of fibrosis/cirrhosis and continued histological improvement in patients with chronic hepatitis B. Hepatology 52: 886-893, 2010.

14. Serpaggi J, Carnot F, Nalpas B, Canioni D, Guéchot J, Lebray P, Vallet-Pichard A, Fontaine H, Bedossa P and Pol S: Direct and indirect evidence for the reversibility of cirrhosis. Hum Pathol 37: 1519-1526, 2006.

15. Bortolotti F and Guido M: Reversal of liver cirrhosis: A desirable clinical outcome and its pathogenic background. J Pediatr Gastroenterol Nutr 44: 401-406, 2007.

16. Desmet VJ: Comments on cirrhosis reversal. Dig Liver Dis 37: 909-916, 2005.

17. Reif S, Lang A, Lindquist JN, Yata Y, Gabele E, Scanga A, Brenner DA and Rippe RA: The role of focal adhesion kinase-phosphatidylinositol 3-kinase-akt signaling in hepatic stellate cell proliferation and type I collagen expression. J Biol Chem 278: 8083-8090, 2003.

18. Wang J, Chu ES, Chen HY, Man K, Go MY, Huang XR, Lan HY, Sung JJ and Yu J: microRNA-29b prevents liver fibrosis by attenuating hepatic stellate cell activation and inducing apoptosis through targeting PI3K/AKT pathway. Oncotarget 6: 7325-7338, 2015.

19. Hua H, Zhu Y and Song YH: Ruscogenin suppressed the hepatocellular carcinoma metastasis via PI3K/Akt/mTOR signaling pathway. Biomed Pharmacother 101: 115-122, 2018.

20. Gäbele E, Reif S, Tsukada S, Bataller R, Yata Y, Morris T, Schrum LW, Brenner DA and Rippe RA: The role of p70S6K in hepatic stellate cell collagen gene expression and cell proliferation. J Biol Chem 280: 13374-13382, 2005.

21. Coffey JC, Wang JH, Smith MJ, Laing A, Bouchier-Hayes D, Cotter TG and Redmond HP: Phosphoinositide 3-kinase accelerates postoperative tumor growth by inhibiting apoptosis and enhancing resistance to chemotherapy-induced apoptosis. Novel role for an old enemy. J Biol Chem 280: 20968-20977, 2005.

22. Cai CX, Buddha H, Castelino-Prabhu S, Zhang Z, Britton RS, Bacon BR and Neuschwander-Tetri BA: Activation of insulinPI3K/Akt-p70S6K pathway in hepatic stellate cells contributes to fibrosis in nonalcoholic steatohepatitis. Dig Dis Sci 62: 968-978, 2017.

23. Patsenker E, Schneider V, Ledermann M, Saegesser H, Dorn C, Hellerbrand $\mathrm{C}$ and Stickel F: Potent antifibrotic activity of mTOR inhibitors sirolimus and everolimus but not of cyclosporine A and tacrolimus in experimental liver fibrosis. J Hepatol 55: 388-398, 2011. 